\title{
Oncolytic viro-chemotherapy exhibits antitumor effect in laryngeal squamous cell carcinoma cells and mouse xenografts
}

This article was published in the following Dove Press journal:

Cancer Management and Research

\author{
Yigang Wang' \\ Binrong Wang' \\ Junnan Liang ${ }^{2}$ \\ Caixia $\mathrm{Cui}^{3}$ \\ Chang Ying' \\ Fang Huang ${ }^{4}$ \\ Buyun $\mathrm{Ma}^{\mathrm{l}}$ \\ Xiumei Zhou' \\ Liang $\mathrm{Chu}^{2}$
}

'College of Life Sciences, Zhejiang Sci-Tech University, Hangzhou 310018 , People's Republic of China; ${ }^{2}$ Hepatic Surgery Center, Tongji Hospital, Tongji Medical College, Huazhong University of Science and Technology, Wuhan 430030, People's Republic of China; ${ }^{3}$ Department of Otorhinolaryngology, Affiliated Hospital of Hangzhou Normal University, Hangzhou 310015, People's Republic of China; ${ }^{4}$ Department of Pathology, Zhejiang Provincal People's Hospital, Hangzhou 310014, People's Republic of China
Correspondence: Liang Chu Hepatic Surgery Center, Tongji Hospital, Tongji Medical College, Huazhong University of Science and Technology, No. 1095 Jie Fang Avenue, Wuhan 430030,

People's Republic of China

Tel +862783662497

Fax +862783662497

Email liangchu@tjh.tjmu.edu.cn
Background: Oncolytic virus can specifically replicate in and then lyse tumor cells, but seldom in normal cells. Further studies have shown the significant therapeutic effect of oncolytic virotherapy combining with other strategies, such as chemo-, radio-, and immunotherapy et al. In this study, we investigated the combinational effect of oncolytic virus ZD55-TRAIL and chemotherapy drug doxorubicin (DOX) on human laryngeal squamous cell carcinoma (LSCC).

Methods: The effect of ZD55-TRAIL combined with DOX on cell growth was assessed in LSCC Hep2 cells and normal cells by MTT assay. Hochest 33342 staining was performed to observe cell morphological changes. Western blot was used to detect the expression of apoptotic activation proteins. The in vivo antitumor efficacy of combination treatment was estimated in laryngeal cancer xenograft models.

Results: The combination of ZD55-TRAIL and DOX exhibited enhanced inhibitory effects on laryngocarcinoma cell growth, and had few side effects to normal cells in vitro. Chemotherapy drug increased the inducement of tumor cell apoptosis mediated by oncolytic virus. In vivo experiment confirmed that the combination treatment significantly inhibited Hep2 laryngocarcinoma xenografts growth in mice.

Conclusion: The oncolytic viro-chemotherapy is a potent therapeutic approach for in vitro cytotoxicity evaluation of Hep2 cells and xenograft growth in vivo.

Keywords: laryngocarcinoma, oncolytic virus, ZD55-TRAIL, doxorubicin

\section{Introduction}

Human laryngeal squamous cell carcinoma (LSCC) is the sixth most common cancer worldwide with high morbidity and mortality, which is a serious threat to human health. ${ }^{1}$ The incidence rate is about $16 \% \sim 40 \%$ of systemic malignant tumors according to the National Cancer Institute Data of Surveillance, Epidemiology, and End Results (SEER). ${ }^{2,3}$ In the United States, >55,000 new cases of LSCC were found each year, and among them 12,000 were dead. ${ }^{2,4}$ Although the development of early detection technique largely improved laryngeal cancer diagnosis and prognosis, it is still hard to eradicate the high-grade laryngeal cancer. ${ }^{5}$ Therefore, novel therapeutic strategies for LSCC are urgently needed.

Recently, oncolytic virotherapy has attracted great attention since oncolytic virus can specifically proliferate in and finally lyse tumor cells. ${ }^{6-8}$ Even a small quantity of oncolytic virus can selectively spread to surrounding tumor cells. ${ }^{9,10}$ Up to date, $>12$ different oncolytic viruses are undergoing Phase I-III clinical trials for treatment of 
various types of cancers, ${ }^{11}$ including adenovirus, poxvirus, herpes simplex virus (HSV), and reovirus. ${ }^{12,13}$ In particular, the first authorized oncolytic virus agent by US Food and Drug Administration, Talimogene laherparepvec (T-VEC), which is a genetically modified HSV delivering granulocyte macrophage colony-stimulating factor (GM-CSF), improved durable response rate in patients with advanced melanoma in a Phase III trial. ${ }^{14}$ Further studies have shown the significant therapeutic effect of oncolytic virotherapy combining with other strategies such as chemo-, radio-, and immunotherapy. ${ }^{15}$

In our previous studies, although oncolytic adenovirus acquired a great achievement focusing on treating solid tumors, ${ }^{16,17}$ the antitumor effect of adenovirus particles alone is limited. It makes us explore more effective regime, such as oncolytic viro-chemotherapy and viroradiotherapy. To be noted, the combined therapy indeed made great progress in preclinical or clinic cancer trials. ${ }^{18}$ The oncolytic adenoviruses include ZD55-TRAIL, H101, AD5/3.2xTyr, ${ }^{19}$ CRAdRGDflt-IL24, ${ }^{20}$ Ad5/5-D24 ${ }^{21}$, and SG600. ${ }^{22}$ ZD55-TRAIL, an E1B55K gene-deleted oncolytic adenovirus harboring tumor necrosis factor-related apoptosis-inducing ligand (TRAIL) constructed by us, exerted efficient cancer therapy effect. ${ }^{23}$

TRAIL is a member of the tumor necrosis factor family that selectively induces apoptosis in tumor cells but not in most normal cells. However, tumor cells represent resistance to TRAIL. As a traditional chemotherapy drug, doxorubicin (DOX) could inhibit tumor cell cycle and induce apoptosis in a wide-broad human tumors, ${ }^{24,25}$ but chemotherapy could cause side effect to normal cells. Therefore, how to improve cell sensitivity to TRAIL and reduce DOX toxic effect is needed to solve. In this study, we investigated the therapeutic effect of ZD55-TRAIL combining with DOX in LSCC. The results showed that the combination treatment effectively inhibited tumor growth both in vitro and in vivo, enhanced tumor cell apoptosis, and decreased the toxicity effect on normal cells in vitro by reducing DOX dosage.

\section{Materials and methods}

\section{Cell lines and viruses}

HEK293 cell line was obtained from Microbix Biosystems Inc. (Toronto, Ontario, Canada). Human normal liver cell line L-02 and human LSCC cell line Hep2 were purchased from the Shanghai Cell Collection (Shanghai, China).
HEK293 and L-02 cells were cultured in DMEM (GIBCO BRL, Grand Island, NY, USA) supplemented with 10\% heat-inactivated FBS (GIBCO BRL). Hep2 cells were cultured in 1640 supplemented with 10\% FBS. All cells were cultured in a $5 \% \mathrm{CO}_{2}$ humidified incubator at $37^{\circ} \mathrm{C}$.

ZD55-TRAIL, ZD55-enhanced green fluorescent protein (EGFP) virus was amplified in HEK293 cells, and the large-scale purification was performed by ultracentrifugation in a cesium chloride gradient, followed by dialysis. Virus titer was determined by TCID50 assay in HEK293 cells. EGFP expression was observed by fluorescence microscope (IX71-22FL/PH, Olympus Corporation, Tokyo, Japan), and the percentage of EGFP-positive cells was measured by flow cytometry.

\section{Comparative replication assay}

To determine the relative replication efficiency of virus, Hep2 cells were infected with ZD55-TRAIL, ZD55TRAIL plus DOX in a humidified chamber at $37^{\circ} \mathrm{C}$ with $5 \% \mathrm{CO}_{2}$ air and maintained for $48 \mathrm{hrs}$. The progeny virus was collected from supernatant for virus titer determination.

\section{Assessment of viral E4 levels}

Total DNA was obtained from each sample using FastPure cell/tissue DNA isolation mini kit (Vazyme, Nanjing, China). E4 gene was measured by quantitative real-time PCR and normalized with a standard. Standard curves were generated by serial dilutions of pAdeasy-1, which is a $33.4 \mathrm{~kb}$ plasmid containing most of the human adenovirus serotype $\mathrm{V}$ genome (ThermoFisher scientific, Waltham, MA, USA). The primers sequences are: E4, forward 5'-CTAACCAGCGTAGCCCCGA-3' and reverse 5'-TGAGCAGCACCTTGCATTTT-3'; GAPDH, forward 5'-ATTCCACGGCACAGTCAAGG-3' and reverse 5'ACATACTCAGCACCAGCATCG-3'. All the reactions were done in triplicates.

\section{Assessment of TRAIL mRNA expression}

Total RNA was extracted from each sample to assess for TRAIL levels using quantitative real-time PCR. TRIzol reagent (ThermoFisher) was used for RNA isolation. Singlestrand cDNA was synthesized using ReverTra Ace qPCR RT Kit (Toyobo, Osaka, Japan), and SuperReal Premix plus (Vazyme) was used for amplification. The primer sequences are: TRAIL, forward 5'-TGCGTGCTGATCGTGATCTTC-3' and reverse 5'-GCTCGTTGGTAAAGTACACGTA-3'. All the reactions were done in triplicates, and the results obtained for TRAIL were normalized to GAPDH. 


\section{Cell viability assay}

Cells were seeded in 96-well plates for $12 \mathrm{hrs}$, and then treated with ZD55-TRAIL, DOX, ZD55-TRAIL plus DOX. At the indicated time points after treatment, the medium was removed and fresh medium containing (MTT, $0.5 \mathrm{mg} / \mathrm{mL}$ ) was added to each well. After $4 \mathrm{hrs}$ in the incubator, the supernatant was removed and $150 \mu \mathrm{L}$ dimethyl sulfoxide was added to each well. Absorbance was read at $490 \mathrm{~nm}$ with a microplate reader (TECAN, Grödig, Austria).

\section{Western blot analysis}

Total proteins were collected by cell lysis buffer (Beyotime, Nantong, China). Protein concentrations were determined by Pierce BCA protein assay kit (ThermoFisher scientific). Proteins were separated by SDS-PAGE on an $8-12 \%$ gel and then transferred to a $0.45 \mu \mathrm{m}$ nitrocellulose membrane (Millipore Corp., Bedford, MA, USA). After being blocked in blocking buffer ( $5 \%$ bovine serum albumin, $10 \mathrm{mmol} / \mathrm{L}$ Tris- $\mathrm{HCl}, \mathrm{pH} 8.0,150 \mathrm{mmol} / \mathrm{L} \mathrm{NaCl}$, and $0.05 \%$ Tween 20 ), the membranes were incubated with primary antibodies and the corresponding secondary fluorescent antibodies (LI-COR Biosciences Inc., Lincoln, NE, USA). The fluorescent signal was detected by the Odyssey infrared imaging system (LICOR Biosciences Inc.). The primary antibody anti-E1A, TRAIL, Bax, and GAPDH were purchased from Santa Cruz Biotechnology (Santa Cruz, CA, USA). XIAP, PARP, and caspase- 8 antibodies were purchased from Cell Signaling Technology (Danvers, MA, USA).

\section{Cytopathic assay}

Hep2 cells were grown in 48-well plates, then treated with ZD55-TRAIL, DOX, ZD55-TRAIL plus DOX. Two days later, cells were exposed to $2 \%$ crystal violet in $20 \%$ methanol for 15 mins and then washed with distilled water and documented by photography. Uninfected cells served as control.

\section{Hochest33342 staining}

Cells were seeded in 6-well plates at a density of $2.5 \times 10^{5}$ per well overnight. $48 \mathrm{hrs}$ after treatment, cells were incubated with $1 \mu \mathrm{g} / \mu \mathrm{L}$ hochest33342 (Beyotime) for 15 mins, washed twice with $\mathrm{ddH}_{2} \mathrm{O}$, and observed under a fluorescence microscope.

\section{Flow cytometric analysis}

Adherent cells were trypsinized and harvested at $48 \mathrm{hrs}$ after treatment. Apoptosis cells were detected using Annexin-V- fluorescein isothiocyanate (FITC) and propidium iodide (PI) double staining following the manufacturer's (Beyotime).

\section{Animal experiments}

4- to 5-week-old female BALB/c nude mice were purchased from the Shanghai Animal Experimental Center of the Chinese Academy of Sciences. The present study was approved by the ethics committee of College of Life Sciences, Zhejiang Sci-Tech University. All procedures in animal experiments were performed according to institutional guidelines and conformed to the National Institutes of Health guidelines on the ethical use of animals. $5 \times 10^{6}$ Hep 2 cells were subcutaneously injected into the right flank of nude mice. When tumor volumes reached $70-100 \mathrm{~mm}^{3}$, the mice were randomly divided into four groups. The mice borne with tumors were treated with ZD55-TRAIL, DOX, ZD55-TRAIL combined with DOX or PBS, respectively. Total $1 \times 10^{9}$ plaque-forming units (PFUs) ZD55-TRAIL were injected into each mouse via an intratumoral manner for three times every other day, while DOX was injected into the peritoneal cavity at a dose of $4 \mathrm{mg} / \mathrm{kg}$ body weight for three consecutive days. Tumor size was measured by a vernier caliper every 3 or 4 days. Tumor volume (V) was calculated according to the formula: $V\left(\mathrm{~mm}^{3}\right)$ $=1 / 2 \times$ length $\times$ width $\times$ width.

\section{H\&E staining and TUNEL assay}

Tissues were fixed in 10\% formalin, dehydrated with graded concentrations of ethanol, and embedded in paraffin wax. The sections were stained with HE for histological analysis. For TUNEL assay, an in situ apoptosis detection kit (Sino-American Biotechnology Co., Luoyang, China) was used.

Briefly, tumor sections were incubated with proteinase K (Merck Co., Darmstadt, Germany) and rinsed with $\mathrm{ddH}_{2} \mathrm{O}$, then dewaxed with dimethylbenzene, and rehydrated with gradient ethanol twice, each for 5 mins. Endogenous peroxidase was blocked with 3\% $\mathrm{H}_{2} \mathrm{O}_{2}$, and sections were incubated with equilibration buffer and terminal deoxynucleotidyl transferase (TdT) enzyme. Finally, the sections were incubated with antidigoxigenin-peroxidase conjugate. Peroxidase activity in each tissue section was shown by the application of DAB (peroxidase substrate kit, Sino-American Biotechnology). Hematoxylin was used as a counterstain. 


\section{Statistical analysis}

The combined effects of DOX and ZD55-TRAIL on cell viability were analyzed with the median-effect methods of Chou and Talalay ${ }^{26}$ using CalcuSyn software (Biosoft, Ferguson, MO, USA). The combination index (CI) values were used to evaluate the interaction between the drug and virus. For the fraction of virus affected combination index (Fa-CI) plot analysis, $\mathrm{CI}<1,=1$, and $>1$ is defined as synergism, additive effect, and antagonism, respectively. The data were confirmed with the isobologram method. ${ }^{27,28}$

The experimental statistical significance was analyzed by Graph Pad 6.0 software and Student's $t$-test. When $p<0.05$, it is considered statistically significant.

\section{Results}

\section{Characterization of ZD55-TRAIL in laryngocarcinoma cells}

Oncolytic viral vector ZD55 was constructed based on adenovirus serotype $\mathrm{V}$ with an E1B 55-kDa gene deletion, enabling selective replication in many types of tumor cells. $^{15}$ ZD55-TRAIL was obtained by inserting TRAIL gene expression frame into the deleted E1B 55-kDa region under the control of human cytomegalovirus immediateearly (CMV-IE) promoter (Figure 1A). Hep2 cells were treated with ZD55-TRAIL, DOX, or the combination for 48 hrs. The combined treatment caused more adenovirus E1A and TRAIL protein expression in Hep2 cells than those in ZD55-TRAIL-infected cells. There was no E1A expression in mock cells and cells treated with DOX (Figure 1B). The ZD55-TRAIL replication capacity was enhanced by DOX (Figure 1C). The DNA level of E4 gene, another early adenoviral gene, was higher in the combination-treatment group than ZD55-TRAIL infected group (Figure 1D).

\section{ZD55-TRAIL exerts synergistic effect with DOX in laryngocarcinoma cells}

Hep2 cells were treated with DOX (from $0.05 \mu \mathrm{mol} / \mathrm{L}$ to $0.8 \mu \mathrm{mol} / \mathrm{L}$ ), ZD55-TRAIL (from $2 \mathrm{MOI}$ to $32 \mathrm{MOI}$ ), or a combination at the constant ratio of 1:40 for $48 \mathrm{hrs}$. The combination of ZD55-TRAIL and DOX displayed an enhanced tumor-killing effect in Hep2 cells (Figure 2A). The survival rate of cells with $0.2,0.7$, and $0.8 \mu \mathrm{mol} / \mathrm{L}$
A

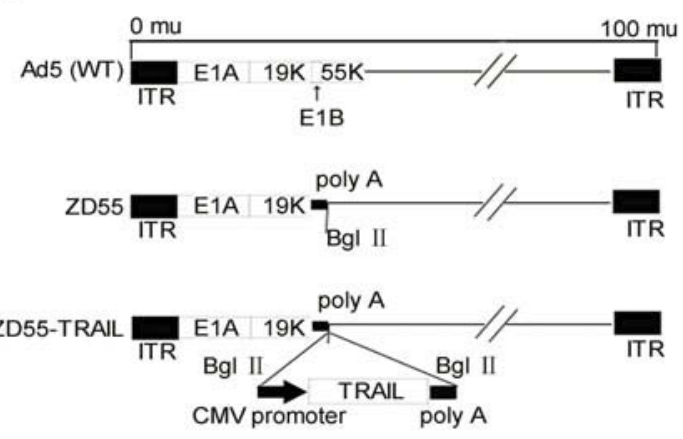

B

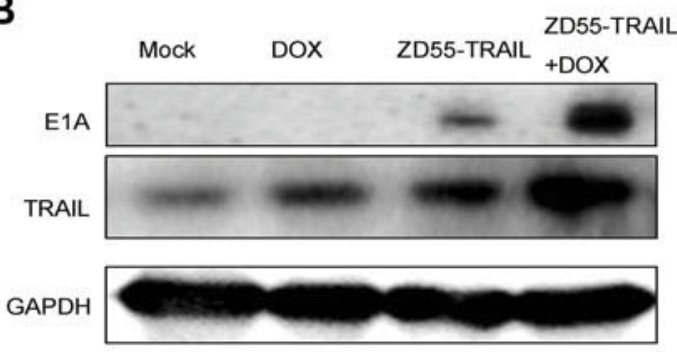

C

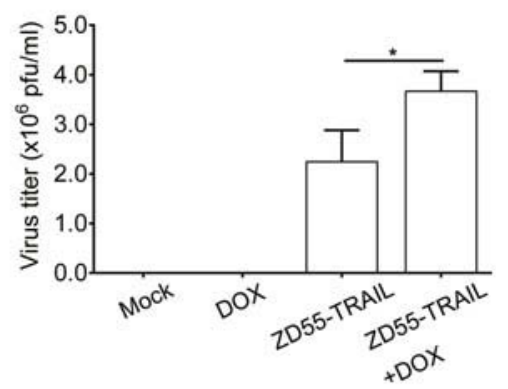

D

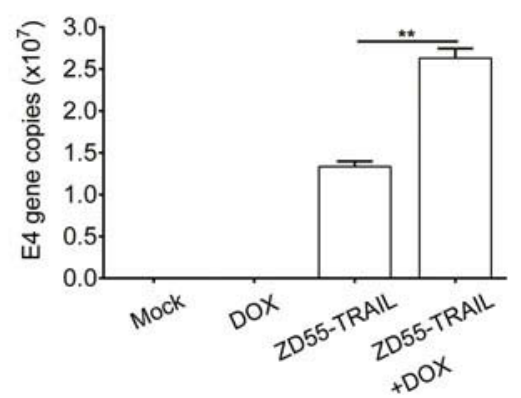

Figure I Characterization of oncolytic adenovirus ZD55-TRAIL. (A) The diagram of adenovirus. WT, wild type. ITR, inverted terminal repeats. (B) Identification of recombinant adenovirus by western blot analysis. ZD55-TRAIL (8MOI), DOX (0.2 $\mu \mathrm{mol} / \mathrm{L})$, or ZD55-TRAIL (8MOI) plus DOX (0.2 $\mu \mathrm{mol} / \mathrm{L})$ were used to treat Hep2 cells for 48 hrs. Collected cell proteins for analyzing the expression of EIA and TRAIL. Untreated cells served as control. GAPDH was used as a protein loading control. (C) Hep2 cells were treated as (B). The progeny virus was collected for virus titer determination. (D) E4 gene copies were determined as a measurement of viral replication. Data are presented in (C, D) as mean \pm SD of three independent experiments. ${ }^{*} p<0.05, * * p<0.01$.

Abbreviations: DOX, doxorubicin; TRAIL, tumor necrosis factor-related apoptosis-inducing ligand. 
A

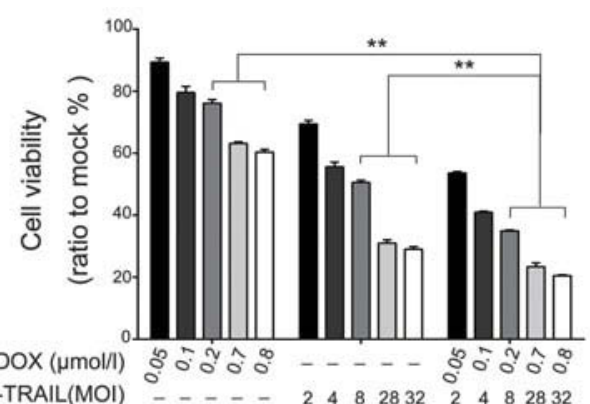

ZD55-TRAIL(MOI)

C

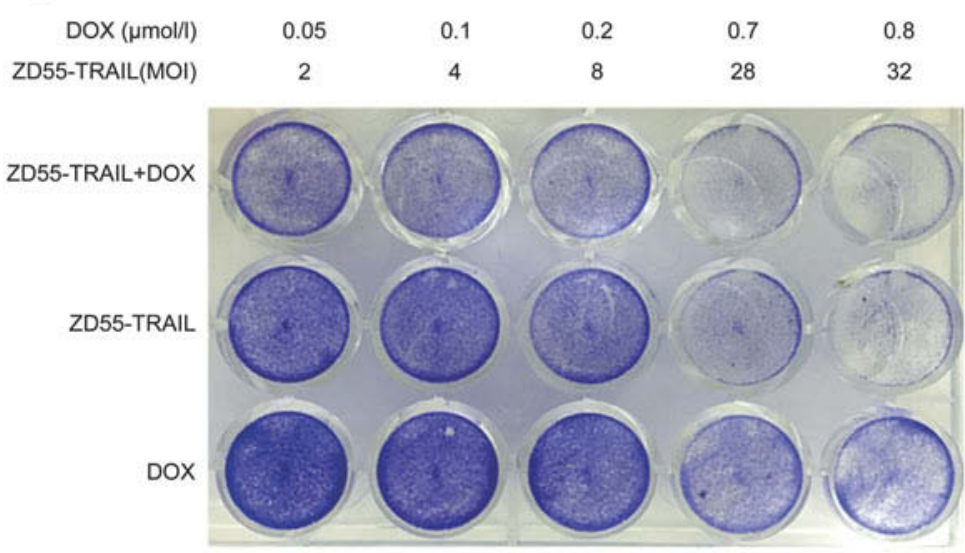

F

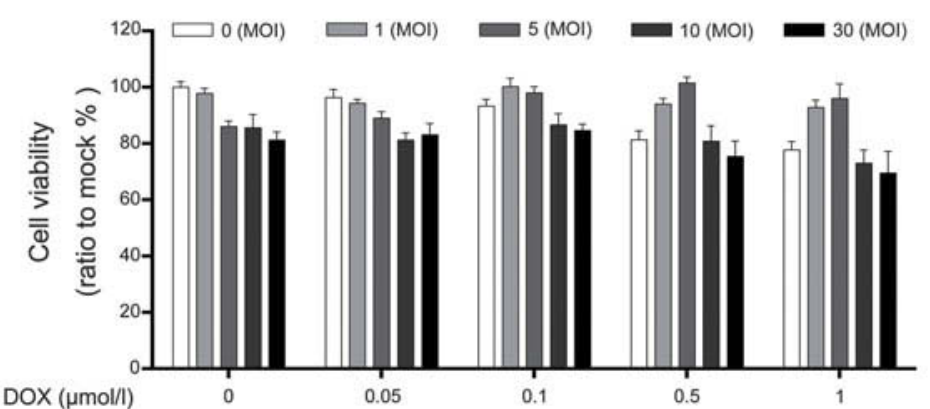

B

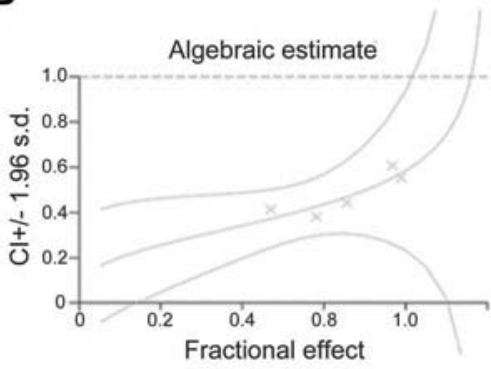

D

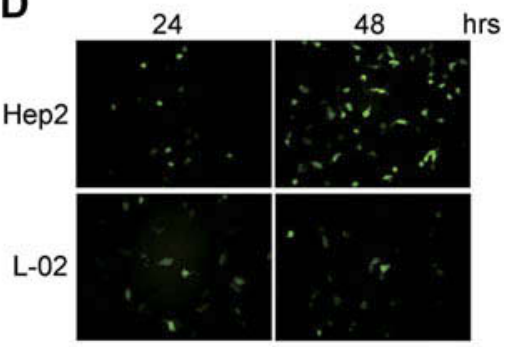

E

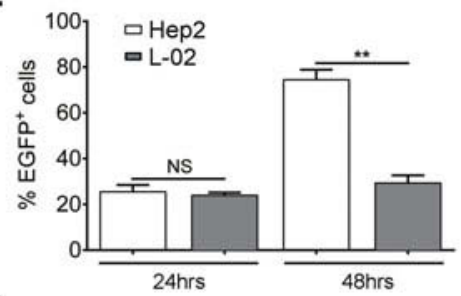

G

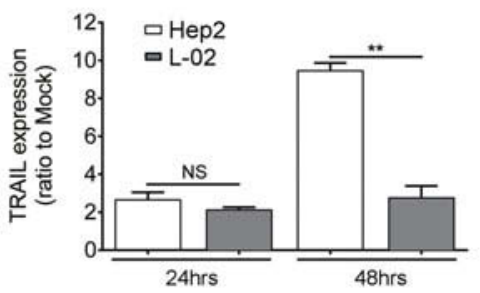

Figure 2 The combination of ZD55-TRAIL and DOX suppressed tumor cell proliferation. (A) Hep2 cells were treated with ZD55-TRAIL, DOX, or ZD55-TRAIL plus DOX for 48 hrs with the indicated dosage. Cell viability was evaluated by the MTT assay. (B) Synergistic effect of ZD55-TRAIL combined with DOX was quantified by combination index $(\mathrm{Cl})$ analysis and expressed as log $(\mathrm{Cl})$ versus fraction affected. $95 \%$ confidence intervals are shown. (C) Hep2 cells were subjected to crystal violet staining for cell viability determination 48 hrs after treatment. (D) Representative fluorescence microscope images of EGFP expression in Hep2 and L-02 cells after I0MOI ZD55-EGFP infection. (E) The percentage of EGFP-positive cells was measured by flow cytometry. (F) L-02 cells were treated with ZD55-TRAIL, DOX, or ZD55-TRAIL plus DOX for $48 \mathrm{hrs}$ with the indicated dosage. Cell viability was determined by the MTT assay. (G) Expression of TRAIL mRNA was detected in Hep2 and L-02 cells using qRT-PCR at the indicated time points after IOMOI ZD55-TRAIL infection. Data are presented in (A, E, F, G) as mean \pm SD of three independent experiments. **p<0.0I, NS, nonsignificant.

DOX treatment was $76.57 \%, 63.01 \%, 59.57 \%$, and $50.54 \%$, $30.95 \%, 28.99 \%$ after $8,28,32$ MOI of ZD55-TRAIL infection, respectively. All were higher than the survival rate of cells with the combination treatment $(34.43 \%$, 23.34\%, and 20.54\%) (Figure 2A). Besides, ZD55-TRAIL plus DOX indicated a synergistic antitumor effect analyzed by the median-effect methods of Chou and Talalay. We evaluated these quantitated data by $\mathrm{Fa}-\mathrm{Cl}$ (Figure 2B). The $\mathrm{X}$ marks represented the $\mathrm{CI}$ values of the combination treatment groups. The CI values were 0.416, 0.381, 0.444, 0.609 , and 0.55 , respectively (Figure $2 \mathrm{~B}$ ). The middle curve line represented the simulated CI values of the combination treatment groups surrounded by two lines of algebraic estimations of the $95 \%$ confidence intervals. All experimental CI values at the tested ratio were significantly $<1$ and between the two confidence lines, indicating synergism of combination treatments. Moreover, the result of crystal violet staining for cell viability determination showed that 
conjugation of DOX with ZD55-TRAIL obviously inhibited cell growth (Figure $2 \mathrm{C}$ ).

\section{Combination of ZD55-TRAIL and DOX does not increase cytotoxicity to normal cells}

The EGFP protein expression was examined in Hep2 and liver normal L-02 cells after ZD55-EGFP virus infection, which is an oncolytic virus carrying EGFP gene. Fluorescence microscope images showed the EGFP expression in both cells and stronger expression in Hep2 cell at $48 \mathrm{hr}$ time point (Figure 2D), consistent with the flow cytometry analysis results (Figure 2E). After confirmation of virus infection, it is necessary to evaluate the toxicity of ZD55-TRAIL and chemotherapeutic agent on normal cells. L-02 cells were treated with ZD55-TRAIL at different MOI, and the cell viability was detected by MTT assay. It is noticeable that the survival rate of L-02 cells kept at $84.42 \%$ after co-treated with the highest dosage of ZD55-TRAIL (30MOI) and DOX (1 $\mu \mathrm{mol} / \mathrm{l})$, indicating the combination treatment caused little toxic effect in L-02 cells (Figure 2F). The mRNA level of TRAIL gene was significantly higher in Hep2 cells than L-02 cells $48 \mathrm{hrs}$ after infection (Figure 2G), suggesting that oncolytic virus could infect but fail to replicate in L-02 cells.

\section{Combination of ZD55-TRAIL and DOX induces cell apoptosis}

We wondered whether the antitumor effect in Hep2 cells was caused by increased cell apoptosis. First, we performed Hoechst 33342 staining to detect the morphological alterations of Hep2 cells after treatment. The results showed that co-treatment led to significant apoptosis with a feature of chromatin condensation, nuclear fragmentation, and apoptotic bodies, compared with ZD55-TRAIL or DOX-alone treated cells (Figure 3A). Annexin-V and PI staining were performed to quantify cell apoptosis. ZD55TRAIL plus DOX caused higher ratio of apoptosis cells (33\%) than ZD55-TRAIL (15.9\%) and DOX (10.2\%) (Figure 3B). Western blot analysis demonstrated that ZD55-TRAIL activated caspase-dependent apoptotic pathway including activation of caspase- 9 , and the activation could be further enhanced by DOX. Simultaneously, we found that co-treatment downregulated the expression of antiapoptosis protein XIAP and upregulated the expression of proapoptotic protein Bax (Figure 3C).

\section{Combination of ZD55-TRAIL and DOX suppresses tumor growth in vivo}

The above in vitro data verified that the combination of ZD55-TRAIL and DOX had synergistic tumor cell-killing effect and induced tumor cell apoptosis. Next, we investigated the therapeutic effects of DOX and ZD55-TRAIL in nude mice. As shown in Figure 4A, the average tumor volume reached about $550 \mathrm{~mm}^{3}$ within 21 days in the control mice receiving PBS treatment, while DOX or ZD55-TRAIL suppressed tumor growth, with average tumor volume $<300$ $\mathrm{mm}^{3}$. ZD55-TRAIL plus DOX treatment exhibited a remarkable tumor growth suppression effect, with average tumor volume $<100 \mathrm{~mm}^{3}$. It is worth mentioning that the xenograft tumors in three of eight mice in the co-treatment group were almost eradicated. We further detected cytopathic effect in vivo. The H\&E staining for the liver sections showed no distinct toxicity in the combinational treatment (Figure 4B). TUNEL analysis indicated that the combination of TRAIL and DOX resulted in more cell apoptosis in tumors than that of ZD55-TRAIL or DOX alone (Figure 4B).

\section{Discussion}

Oncolytic virotherapy has shown promising therapeutic effects. ${ }^{29}$ The prominent advantage of this approach is that, with adding a small amount of viruses, oncolytic effects can selectively initiate anticancer action and spread to the surrounding regions. We constructed oncolytic viral vector ZD55, which is similar to ONYX-015 with an E1B 55-kDa gene deletion. The difference between them is that ZD55 has a multiple clone site and can deliver genes. ${ }^{30}$ In our previous studies, ZD55 delivered genes including TRAIL, IL24, Smac, and XAF1. and exhibited efficient tumorinhibition capability. ${ }^{31,32}$

TRAIL selectively induces apoptosis in various tumor cells and causes few toxicity to normal cells. ${ }^{33}$ TRAIL recognizes, binds with death receptors (DR4, DR5), ${ }^{34}$ and then initiates the apoptosis signal transduction pathway. ${ }^{35}$ It has been reported that oncolytic virus ZD55-TRAIL selectively replicated in tumor cells, and the exogenous TRAIL gene did not attenuate the replication ability of the oncolytic virus. ${ }^{18}$ However, there are several disadvantages in cancer therapy with ZD55-TRAIL, such as tumor resistance, host immune response, neutralized antibody, and unable to eliminate high-malignant tumors. ${ }^{36}$

Chemotherapeutic drugs, such as DOX, are used as an auxiliary therapeutic method for surgery in treating 
A

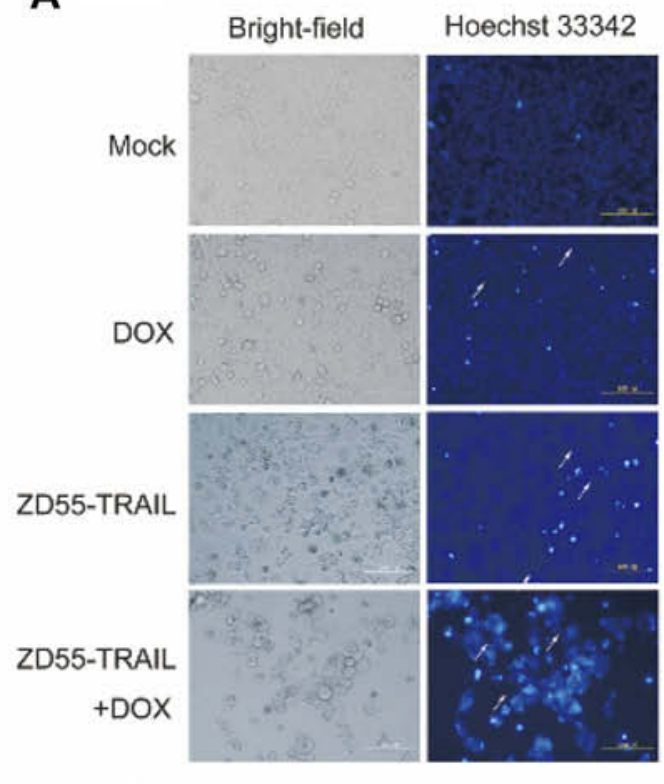

C

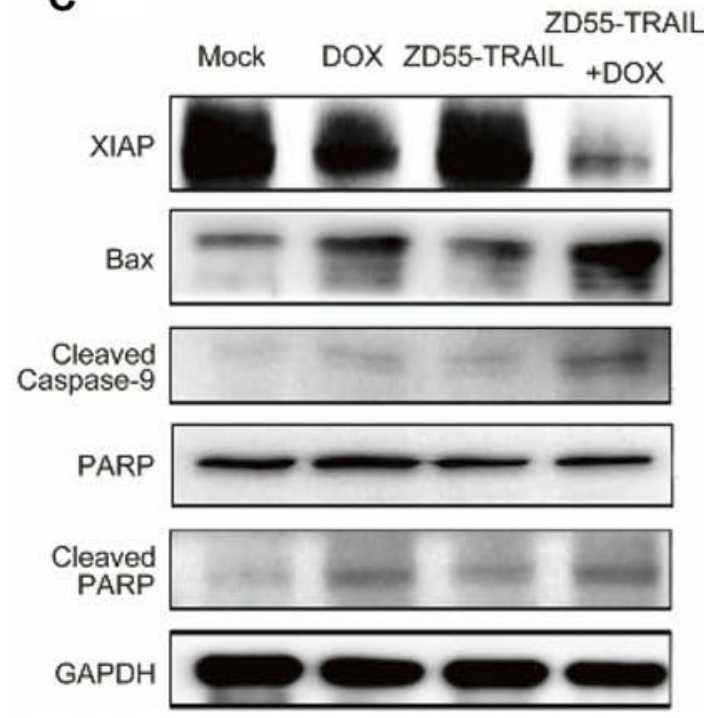

B
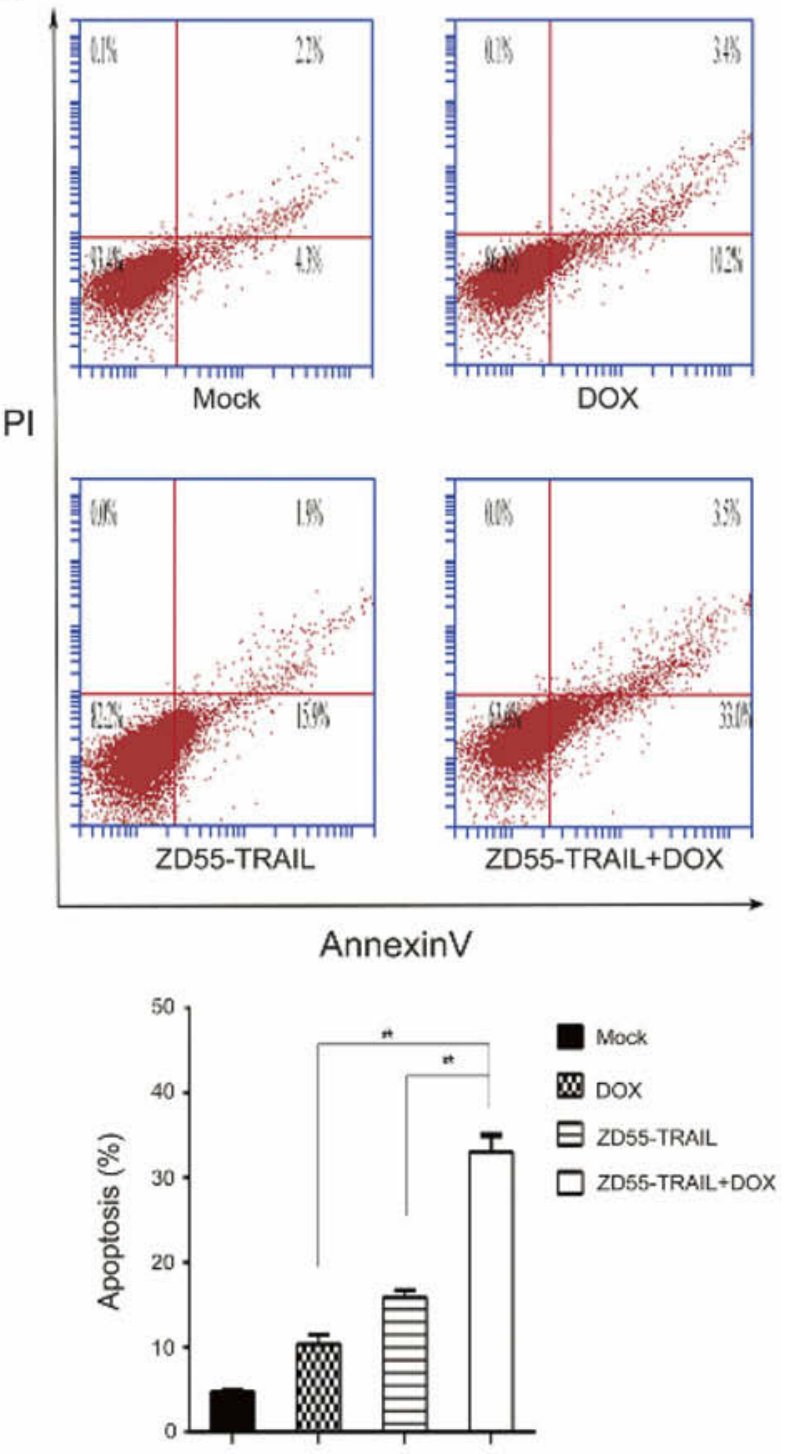

Figure 3 DOX enhanced ZD55-TRAIL-induced apoptosis in Hep2 cells. (A) Hep2 cells were infected with ZD55-TRAIL (8MOI), DOX (0.2 $\mu$ mol/L), or ZD55-TRAIL (8MOI) plus DOX $(0.2 \mu \mathrm{mol} / \mathrm{l}) .48 \mathrm{hrs}$ later, Hep2 cells were stained with Hoechst 33342 . The white arrows indicated apoptotic cells. Original magnification, $200 \times$. (B) 48 hrs after treatment, the apoptosis of Hep2 cells was measured by flow cytometry. ${ }^{* *} p<0.01$. (C) Activation of caspase-mediated apoptosis signaling pathway. Cells were treated as mentioned in (A) for 48 hrs. The expression of the indicated proteins was detected by western blot. GAPDH was used as a loading control.

malignancies. ${ }^{37}$ However, the severe side effects of highdose DOX are essential obstacles. The combination strategies with oncolytic virus and chemotherapy drugs are developed for cancer therapy. In order to obtain an enhanced or synergic therapeutic effect, it is essential to select chemotherapeutic drugs and oncolytic viruses with complementary mechanisms. Studies indicated that chemotherapy drugs upregulated death receptors (DR4 or DR5) or activated intracellular signaling pathways of
TRAIL, suggesting that chemotherapy drugs may sensitize tumor cells to TRAIL-mediated apoptosis. ${ }^{35,38}$

Virus replication and diffusion are restricted in animal studies and clinical trials, especially when the objectives carry tumors with large masses. ${ }^{39-41}$ It is difficult for viruses to penetrate massive tumors, and it may be the reason for disappointing therapeutic outcomes. Therefore, exploring novel tumor biomarkers or therapeutic targets is crucial for oncolytic virus-mediated laryngeal therapy. ${ }^{42-44}$ 

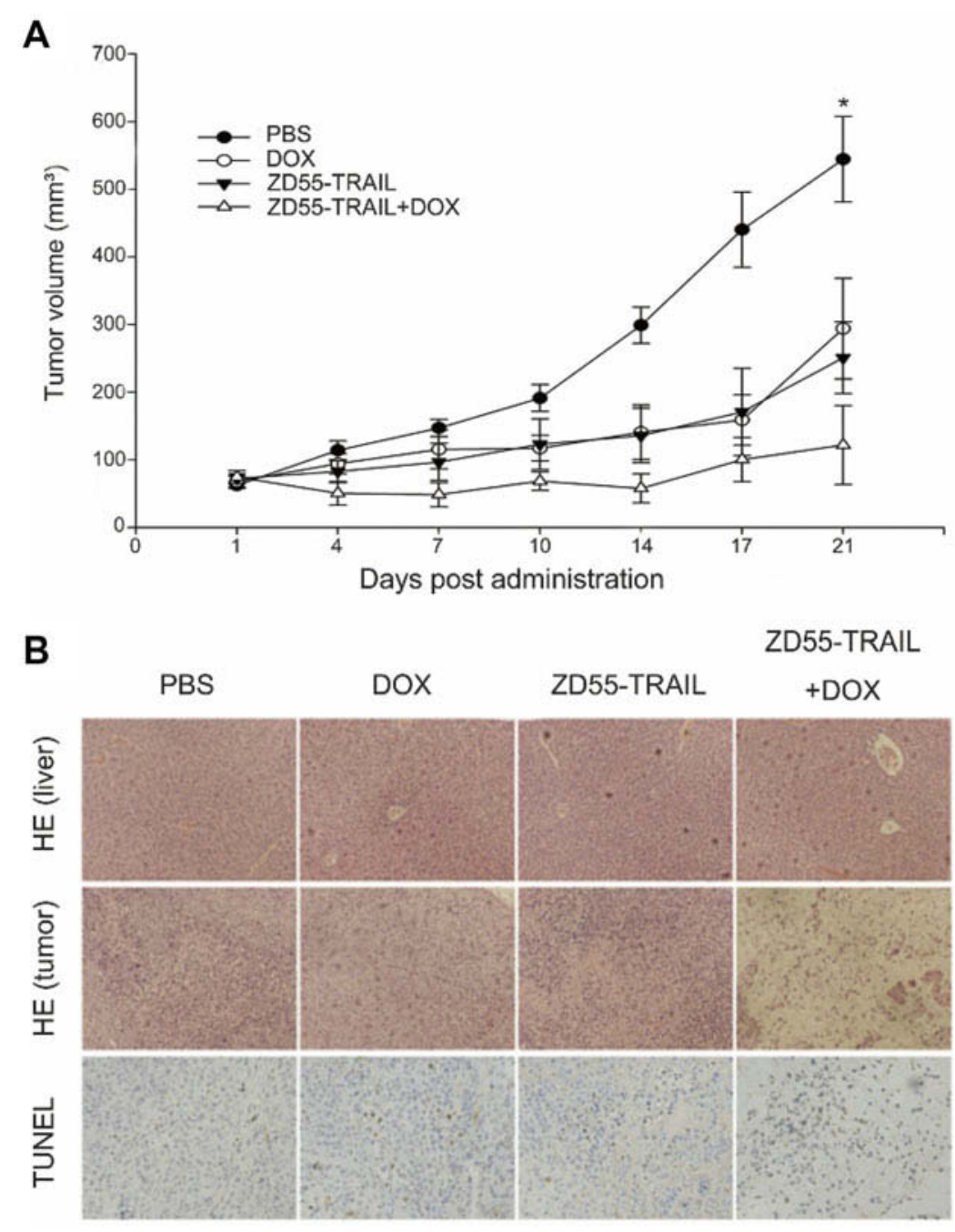

Figure 4 Antitumor effect of DOX and ZD55-TRAIL in Hep2 xenografts. Mice were treated with an intratumoral injection of PBS, DOX, ZD55-TRAIL, or ZD55-TRAIL followed by DOX. (A) Tumor volume was measured. ${ }^{*} p<0.05$. (B) Subcutaneous Hep2 tumors and liver tissues were performed for HE staining analysis (the upper two rows, original magnifcation: 100x). The lower row showed the cell apoptosis in tumor tissues by TUNEL assay (original magnification: 200x).

In the present study, we confirmed the enhanced inhibitory effect of ZD55-TRAIL and DOX in Hep2 cells in vitro and in vivo. The results indicated that ZD55-TRAIL not only replicated, but also expressed TRAIL gene efficiently in Hep2 cells (Figures 1B and 2G). Notably, the addition of DOX was able to enhance the virus replication and TRAIL expression, and resulted in synergic inhibitory effects on the proliferation of Hep2 cells (Figures 1B-D and 2A-C). Besides, ZD55-TRAIL combined with DOX did not increase toxicity in normal liver cells in vitro (Figure 2F). Further, combination treatment induced distinct apoptosis in Hep2 cells compared with individual treatment (Figure 3).

\section{Conclusion}

The oncolytic viro-chemotherapy combining ZD55TRAIL and DOX exerted antitumor effect in cells and in tumor xenografts in mice, indicating that the combined treatment could represent a rational approach for laryngeal cancer therapy.

\section{Ethical approval}

All applicable international, national, and/or institutional guidelines for the care and use of animals were followed.

\section{Acknowledgments}

This work was supported by National Natural Science Fund (31671348, 31301064, 81573000), Zhejiang Provincial Natural Science Foundation of China (No. LY16H160056), Technology bureau of Hangzhou (No. 20130733Q36), the Grant for 521 talent project of ZSTU and Academician workstation of Zhejiang Province. 


\section{Disclosure}

The authors report no conflicts of interest in this work.

\section{References}

1. Vokes EE, Weichselbaum RR, Lippman SM, Hong WK. Head and neck cancer. $N$ Engl J Med. 1993;328(3):184-194. doi:10.1056/ NEJM199301213280306

2. Siegel RL, Miller KD, Jemal A. Cancer statistics, 2017. CA Cancer J Clin. 2017;67(1):7-30. doi:10.3322/caac.21387

3. Steuer CE, El-Deiry M, Parks JR, Higgins KA, Saba NF. An update on larynx cancer. CA Cancer J Clin. 2017;67(1):31-50. doi:10.3322/ caac. 21386

4. Kiang A, Yu MA, Ongkeko WM. Progress and pitfalls in the identification of cancer stem cell-targeting therapies in head and neck squamous cell carcinoma. Curr Med Chem. 2012;19(35):6056-6064.

5. Jiao J, Qin Z, Li S, Liu H, Lu Z. Potential role of Notch1 signaling pathway in laryngeal squamous cell carcinoma cell line Hep-2 involving proliferation inhibition, cell cycle arrest, cell apoptosis, and cell migration. Oncol Rep. 2009;22(4):815-823.

6. Breitbach CJ, Burke J, Jonker D, et al. Intravenous delivery of a multi-mechanistic cancer-targeted oncolytic poxvirus in humans. Nature. 2011;477(7362):99-102. doi:10.1038/nature10358

7. He G, Lei W, Wang S, et al. Overexpression of tumor suppressor TSLC1 by a survivin-regulated oncolytic adenovirus significantly inhibits hepatocellular carcinoma growth. $J$ Cancer Res Clin Oncol. 2012;138(4):657-670. doi:10.1007/s00432-011-1138-2

8. Wang Y, Liu T, Huang P, et al. A novel Golgi protein (GOLPH2)regulated oncolytic adenovirus exhibits potent antitumor efficacy in hepatocellular carcinoma. Oncotarget. 2015;6(15):13564-13578. doi:10.18632/oncotarget.3769

9. Parker JN, Bauer DF, Cody JJ, Markert JM. Oncolytic viral therapy of malignant glioma. Neurotherapeutics. 2009;6(3):558-569. doi:10.1016/j.nurt.2009.04.011

10. Wold WS, Toth K. Chapter three-Syrian hamster as an animal model to study oncolytic adenoviruses and to evaluate the efficacy of antiviral compounds. Adv Cancer Res. 2012;115:69-92. doi:10.1016/ B978-0-12-398342-8.00003-3

11. Li J, Bonifati S, Hristov G, et al. Synergistic combination of valproic acid and oncolytic parvovirus $\mathrm{H}-1 \mathrm{PV}$ as a potential therapy against cervical and pancreatic carcinomas. EMBO Mol Med. 2013;5 (10):1537-1555. doi:10.1002/emmm.201302796

12. Zhang R, Zhang X, Ma B, et al. Enhanced antitumor effect of combining TRAIL and MnSOD mediated by CEA-controlled oncolytic adenovirus in lung cancer. Cancer Gene Ther. 2016;23(6):168177. doi:10.1038/cgt.2016.11

13. Huang F, Wang BR, Wu YQ, Wang FC, Zhang J, Wang YG. Oncolytic viruses against cancer stem cells: a promising approach for gastrointestinal cancer. World J Gastroenterol. 2016;22(35):79998009. doi:10.3748/wjg.v22.i35.7999

14. Kohlhapp FJ, Zloza A, Kaufman HL. Talimogene laherparepvec (TVEC) as cancer immunotherapy. Drugs Today (Barc). 2015;51 (9):549-558. doi:10.1358/dot.2015.51.9.2383044

15. Ma B, Wang Y, Zhou X, et al. Synergistic suppression effect on tumor growth of hepatocellular carcinoma by combining oncolytic adenovirus carrying XAF1 with cisplatin. $J$ Cancer Res Clin Oncol. 2015;141(3):419-429. doi:10.1007/s00432-014-1835-8

16. Lei W, Liu HB, Wang SB, et al. Tumor suppressor in lung cancer-1 (TSLC1) mediated by dual-regulated oncolytic adenovirus exerts specific antitumor actions in a mouse model. Acta Pharmacol Sin. 2013;34(4):531-540. doi:10.1038/aps.2012.196

17. Zhang X, Meng S, Zhang R, et al. GP73-regulated oncolytic adenoviruses possess potent killing effect on human liver cancer stem-like cells. Oncotarget. 2016;7(20):29346-29358. doi:10.18632/oncotarget.8830
18. Liu T, Han HF, Bu-Yun MA, Yang YQ, Zhuo LY. Novel synergistic anti-tumor effects of DOX with oncolytic adenovirus ZD55-TRAIL on hepatocellular carcinoma cells. China Biotechnol. 2014;34(2):26-33.

19. Quirin C, Mainka A, Hesse A, Nettelbeck DM. Combining adenoviral oncolysis with temozolomide improves cell killing of melanoma cells. Int J Cancer. 2007;121(12):2801-2807. doi:10.1002/ijc.23052

20. Kaliberova LN, Krendelchtchikova V, Harmon DK, et al. CRAdRGDflt-IL24 virotherapy in combination with chemotherapy of experimental glioma. Cancer Gene Ther. 2009;16(10):794-805. doi:10.1038/cgt.2009.23

21. Kangasniemi L, Parviainen S, Pisto T, et al. Effects of capsid-modified oncolytic adenoviruses and their combinations with gemcitabine or silica gel on pancreatic cancer. Int J Cancer. 2012;131(1):253-263. doi:10.1002/ijc. 26370

22. Song X, Wang H, Jia R, et al. Combined treatment with an oncolytic adenovirus and antitumor activity of vincristine against retinoblastoma cells. Int J Mol Sci. 2012;13(9):10736-10749. doi:10.3390/ ijms130910736

23. Wang SB, Tan Y, Lei W, et al. Complete eradication of xenograft hepatoma by oncolytic adenovirus ZD55 harboring TRAIL-IETDSmac gene with broad antitumor effect. Hum Gene Ther. 2012;23 (9):992-1002. doi:10.1089/hum.2011.159

24. Komdeur R, Meijer C, Van Zweeden M, et al. Doxorubicin potentiates TRAIL cytotoxicity and apoptosis and can overcome TRAIL-resistance in rhabdomyosarcoma cells. Int J Oncol. 2004;25(3):677-684.

25. Gish RG, Porta C, Lazar L, et al. Phase III randomized controlled trial comparing the survival of patients with unresectable hepatocellular carcinoma treated with nolatrexed or doxorubicin. J Clin Oncol. 2007;25(21):3069-3075. doi:10.1200/JCO.2006.08.4046

26. Chou TC, Talalay P. Quantitative analysis of dose-effect relationships: the combined effects of multiple drugs or enzyme inhibitors. Adv Enzyme Regul. 1994;22(84):27-55. doi:10.1016/0065-2571(84)90007-4

27. Steel GG, Peckham MJ. Exploitable mechanisms in combined radiotherapy-chemotherapy: the concept of additivity. Int J Radiat Oncol Biol Phys. 1979;5(1):85-91.

28. Niu C, Bao H, Tolstykh T, et al. Evaluation of the in vitro anti-HBV activity of clevudine in combination with other nucleoside/nucleotide inhibitors. Antivir Ther. 2010;15(3):401-412. doi:10.3851/IMP1541

29. Vaha-Koskela MJ, Heikkila JE, Hinkkanen AE. Oncolytic viruses in cancer therapy. Cancer Lett. 2007;254(2):178-216. doi:10.1016/j. canlet.2007.02.002

30. Zhao L, Gu J, Dong A, et al. Potent antitumor activity of oncolytic adenovirus expressing mda- 7/IL-24 for colorectal cancer. Hum Gene Ther. 2005;16(7):845-858. doi:10.1089/hum.2005.16.845

31. Pan Q, Liu B, Liu J, Cai R, Wang Y, Qian C. Synergistic induction of tumor cell death by combining cisplatin with an oncolytic adenovirus carrying TRAIL. Mol Cell Biochem. 2007;304(1-2):315-323. doi:10.1007/s11010-007-9514-6

32. Qi R, Gu J, Zhang Z, et al. Potent antitumor efficacy of XAF1 delivered by conditionally replicative adenovirus vector via caspase-independent apoptosis. Cancer Gene Ther. 2007;14(1):82-90. doi:10.1038/sj.cgt. 7700992

33. Walczak H, Miller RE, Ariail K, et al. Tumoricidal activity of tumor necrosis factor-related apoptosis-inducing ligand in vivo. Nat Med. 1999;5(2):157-163. doi:10.1038/5517

34. Oikonomou E, Pintzas A. The TRAIL of oncogenes to apoptosis. Biofactors. 2013;39(4):343-354. doi:10.1002/biof.1112

35. Dai Y, Liu M, Tang W, et al. A Smac-mimetic sensitizes prostate cancer cells to TRAIL-induced apoptosis via modulating both IAPs and NFkappaB. BMC Cancer. 2009;9(392). doi:10.1186/1471-2407-9-392.

36. Reeh M, Bockhorn M, Gorgens D, et al. Presence of the coxsackievirus and adenovirus receptor (CAR) in human neoplasms: a multitumour array analysis. $B r J$ Cancer. 2013;109(7):1848-1858. doi:10.1038/bjc.2013.509

37. Fillastre JP, Raguenez-Viotte G. Cisplatin nephrotoxicity. Toxicol Lett. 1989;46(1-3):163-175. 
38. Wiley SR, Schooley K, Smolak PJ, et al. Identification and characterization of a new member of the TNF family that induces apoptosis. Immunity. 1995;3(6):673-682.

39. Zhao T, Rao XM, Xie X, et al. Adenovirus with insertion-mutated E1A selectively propagates in liver cancer cells and destroys tumors in vivo. Cancer Res. 2003;63(12):3073-3078.

40. Kirn D. Clinical research results with d11520 (Onyx-015), a replication-selective adenovirus for the treatment of cancer: what have we learned? Gene Ther. 2001;8(2):89-98. doi:10.1038/sj.gt.3301377

41. Yamamoto M, Curiel DT. Current issues and future directions of oncolytic adenoviruses. Mol Ther. 2010;18(2):243-250. doi:10.1038/mt.2009.266
42. Ding $X$, Ding J, Ning J, et al. Circulating microRNA-122 as a potential biomarker for liver injury. Mol Med Rep. 2012;5(6):14281432. doi: $10.3892 / \mathrm{mmr} .2012 .838$

43. Wang F, Zheng Z, Guo J, Ding X. Correlation and quantitation of microRNA aberrant expression in tissues and sera from patients with breast tumor. Gynecol Oncol. 2010;119(3):586-593. doi:10.1016/j. ygyno.2010.07.021

44. Zhang S, Zhu C, Zhu L, et al. Oncogenicity of the transcription factor SOX8 in hepatocellular carcinoma. Med Oncol. 2014;31(4):918. doi:10.1007/s12032-014-0374-0

\section{Publish your work in this journal}

Cancer Management and Research is an international, peer-reviewed open access journal focusing on cancer research and the optimal use of preventative and integrated treatment interventions to achieve improved outcomes, enhanced survival and quality of life for the cancer patient.
The manuscript management system is completely online and includes a very quick and fair peer-review system, which is all easy to use. Visit http://www.dovepress.com/testimonials.php to read real quotes from published authors. 